University of Wollongong

Research Online

Faculty of Engineering - Papers (Archive)

Faculty of Engineering and Information

Sciences

$1-1-2006$

\title{
Fiber-optic temperature sensor based on interference of selective higher- order modes
}

Enbang Li

enbang@uow.edu.au

X L Wang

Chao Zhang

University of Wollongong, czhang@uow.edu.au

Follow this and additional works at: https://ro.uow.edu.au/engpapers

Part of the Engineering Commons

https://ro.uow.edu.au/engpapers/3023

\section{Recommended Citation}

Li, Enbang; Wang, X L; and Zhang, Chao: Fiber-optic temperature sensor based on interference of selective higher-order modes 2006, 091119-1-091119-3.

https://ro.uow.edu.au/engpapers/3023

Research Online is the open access institutional repository for the University of Wollongong. For further information contact the UOW Library: research-pubs@uow.edu.au 


\title{
Fiber-optic temperature sensor based on interference of selective higher-order modes
}

\author{
Enbang $\mathrm{Li}^{\mathrm{a})}$ \\ College of Precision Instrument and Optoelectronics Engineering, Tianjin University, Tianjin 300072, \\ People's Republic of China \\ Xiaolin Wang \\ Institute for Superconducting and Electronic Materials, Faculty of Engineering, University of Wollongong, \\ New South Wales 2522, Australia \\ Chao Zhang \\ School of Engineering Physics, University of Wollongong, New South Wales 2522, Australia
}

(Received 26 April 2006; accepted 3 August 2006; published online 31 August 2006)

\begin{abstract}
A fiber-optic temperature sensor based on the interference of selective higher-order modes in circular optical fibers is described. The authors demonstrate that by coupling the $\mathrm{LP}_{01}$ mode in a standard single-mode fiber to the $\mathrm{LP}_{0 m}$ modes in a multimode fiber, and utilizing the interference of the higher-order modes, a fiber-optic temperature sensor which has an extremely simple structure and is suitable for high-temperature measurements can be constructed. The sensing principle, temperature measurement experiments, and results are presented. (C) 2006 American Institute of Physics. [DOI: 10.1063/1.2344835]
\end{abstract}

Fiber-optic temperature sensors offer unique advantages, such as immunity to electromagnetic interferences, stability, repeatability, durability against harsh environments, high sensitivity, high resolution, and fast response. Therefore, optical fiber sensors have been widely adopted and applied in different areas for temperature measurements. ${ }^{1,2}$ Among the different types of temperature sensors, those based on the fiber Bragg grating (FBG) technique have attracted much attention since FBG sensors have the so-called wavelength encoding and wavelength multiplexing features. ${ }^{2-4}$ Determined by the thermal stability of normal FBGs (type I gratings) in which positive refractive index modulations are induced by moderate UV exposures, ${ }^{2,5}$ temperature sensors with type I gratings can normally be used for relatively low temperature (below $200{ }^{\circ} \mathrm{C}$ ) measurements. By utilizing type II or type IIA gratings in which intensive UV exposures cause negative index changes or physical damages, ${ }^{6,7}$ the measurable temperature range can be extended to over $500{ }^{\circ} \mathrm{C}$. Recently, a surface relief FBG sensor, which was fabricated by etching a grating into the flat side of a D-shaped optical fiber, has been used for high-temperature $\left(1100{ }^{\circ} \mathrm{C}\right)$ sensing. $^{8}$ Since the grating is formed by physically changing the fiber geometry, it will not be erased under high temperatures. However, the fabrication process of the surface relief grating is rather complex, and the grating is fragile.

In this letter, we present a fiber-optic temperature sensor by using the interference of selective higher-order modes in circular optical fibers. The proposed temperature sensors have the features similar to those of FBG sensors, such as wavelength encoding and wavelength multiplexing capabilities, and more importantly have an extremely simple structure and the potential for high-temperature measurements.

When a single-mode fiber (SMF) is connected to a multimode fiber (MMF), different modes can be excited in the

${ }^{a)}$ Electronic mail: enbang@tju.edu.cn
MMF and supported by the MMF. Suppose that both the SMF and the MMF have circular cross sections and stepindex distributions. Under these conditions, only linearly polarized radial modes, i.e., $\mathrm{LP}_{0 m}$ ( $m$ is a positive integer), will be excited and transmitted in the MMF. The power of the $\mathrm{LP}_{0 m}$ mode in the MMF is determined by the coupling coefficient from the $\mathrm{LP}_{01}$ mode in the $\mathrm{SMF}$ to the particular mode in the MMF, and is a function of fiber parameters.

Suppose that the length of the MMF is $L$. At the exit end of the MMF, the phase difference between two modes, $\mathrm{LP}_{0 m}$ and $\mathrm{LP}_{0 n}$, is determined by the length of the MMF and the difference of the longitudinal propagation constants of the two modes, $\beta_{m}-\beta_{n}$, which can be expressed as ${ }^{9,10}$

$$
\beta_{m}-\beta_{n}=\frac{u_{m}^{2}-u_{n}^{2}}{2 k a^{2} n_{\mathrm{co}}}
$$

where $u_{m}=\pi(m-1 / 4)$ and $u_{n}=\pi(n-1 / 4)$ are the roots of the Bessel function of zero order, $a$ is the core radius of the MMF, $k=2 \pi / \lambda$ is the free-space wave number, and $n_{\text {co }}$ is the refractive index of the fiber core.

Interferences among different modes happen at the exit end of the MMF. The condition for a constructive interference between $\mathrm{LP}_{0 m}$ and $\mathrm{LP}_{0 n}$ is

$$
\left(\beta_{m}-\beta_{n}\right) L=2 \pi N
$$

where $N$ is an integer.

From Eqs. (1) and (2), we can obtain the wavelengths at which maximum interferences happen, that is,

$$
\lambda=\frac{16 n_{\mathrm{co}} a^{2} N}{(m-n)[2(m+n)-1] L} \quad(m>n) .
$$

The wavelength spacing between two adjacent maxima (or minima) is 


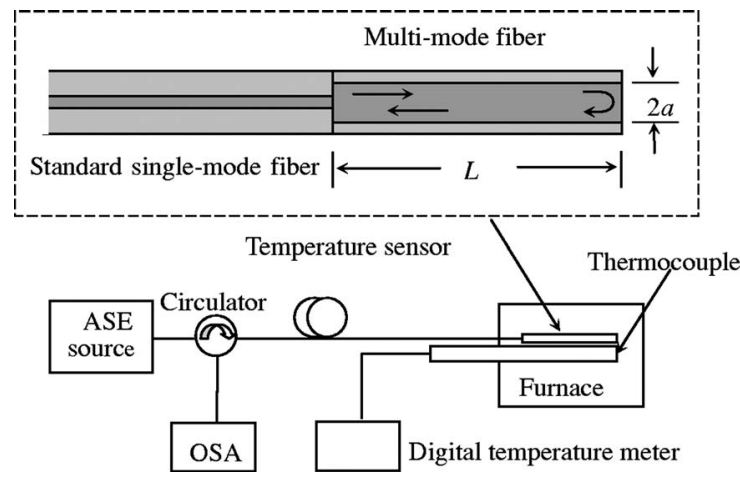

FIG. 1. Schematic diagram of the experimental setup. The inset shows the structure and parameters of the developed temperature sensor.

$$
\lambda_{N}-\lambda_{N-1}=\frac{16 n_{\mathrm{co}} a^{2}}{(m-n)[2(m+n)-1] L} \quad(m>n) .
$$

Since the fiber core refractive index, core radius, and fiber length are dependent on the temperature the MMF is exposed to, the wavelength given by Eq. (3) is also a function of temperature.

From Eq. (3), we can obtain the relative wavelength variation caused by a temperature change, which can be expressed as

$$
\frac{\Delta \lambda}{\lambda}=\frac{\partial n_{c o}}{n_{c o} \partial T} \Delta T+2 \frac{\partial a}{a \partial T} \Delta T-\frac{\partial L}{L \partial T} \Delta T=(\alpha+\xi) \Delta T,
$$

where $\alpha$ and $\xi$ are, respectively, the coefficient of thermal expansion and the thermo-optic coefficient of the MMF material. It can be seen clearly from Eq. (5) that temperature can be measured by detecting the wavelength at which a maximum or minimum interference occurs. In other words, the proposed sensing technique has the wavelength encoding and multiplexing features. It is also obvious that the temperature sensitivity is the same as that of a FBG temperature sensor. $^{2-4}$

A schematic diagram showing the structure and parameters of the temperature sensor is depicted in the inset of Fig. 1. The sensor was fabricated by connecting a $50 \mathrm{~mm}$ long MMF with a core radius of $52.5 \mu \mathrm{m}$ and a cladding radius of $62.5 \mu \mathrm{m}$ to a standard communications SMF by fusion splicing. Using the parameters of the fibers employed, we calculated the coupling coefficients for different LP modes and the results showed that, among the modes excited in the MMF, the $\mathrm{LP}_{06}$ and $\mathrm{LP}_{07}$ modes have the largest coupling coefficients. In order to make a single-ended sensor, we cleaved the free end of the MMF to achieve a surface perpendicular to the fiber axis. This end surface served as a mirror reflecting the light reaching that surface back to the MMF. At the interface between the SMF and the MMF, the modes in the MMF are coupled to the $\mathrm{LP}_{01}$ mode in the SMF.

In an experimental work using the fabricated sensor, the reflected light was separated from the incident light by a three-port optical circulator and transmitted to an optical spectrum analyzer (OSA), as shown in Fig. 1. Using an ASE broadband source with a power of $5 \mathrm{~mW}$, a central wavelength of $1550 \mathrm{~nm}$, and a bandwidth of about $30 \mathrm{~nm}$, we recorded the reflected interference spectra. Shown in Fig. 2 is a spectrum recorded at room temperature $\left(23{ }^{\circ} \mathrm{C}\right)$, from which we can see two dips separated by $24.56 \mathrm{~nm}$. We believe that it was produced by destructive interference be-

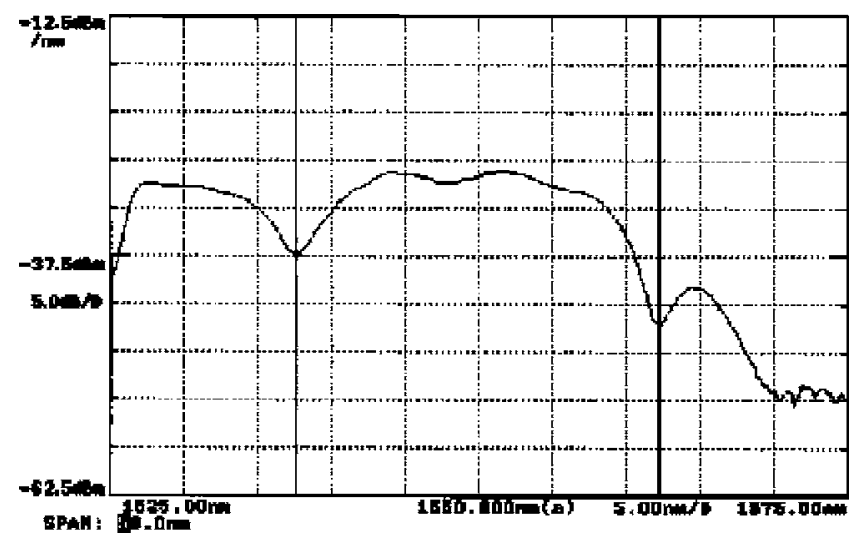

FIG. 2. Measured reflection spectrum of a temperature sensor with a $50 \mathrm{~mm}$ long MMF. The marked dips locate at 1537.587 and $1562.144 \mathrm{~nm}$, respectively.

tween the $\mathrm{LP}_{06}$ and $\mathrm{LP}_{07}$ modes in the MMF. To confirm this, we calculated the wavelength spacing by using Eq. (4) and the following parameters: $L=100 \mathrm{~mm}$ (twice the MMF length), $m=7, n=6, a=52.5 \mu \mathrm{m}$, and $n_{\mathrm{co}}=1.4662$. The calculated wavelength spacing is $25.86 \mathrm{~nm}$, which is close to the measured value $(24.56 \mathrm{~nm})$.

In order to test the ability and performance of the fiber sensor for temperature measurements, we attached the fabricated sensor to a thermocouple, which was connected to a digital temperature meter, and inserted them into a furnace (Thermolyne 48000). The spectra were recorded by the OSA and the wavelengths of the dips on the spectra were measured while the furnace temperatures were changed and measured by the thermocouple. Shown in Fig. 3 are the measured wavelength shifts at different temperatures. It can be seen from Fig. 3 that in a temperature range of $100-750{ }^{\circ} \mathrm{C}$, the wavelength shift increases linearly with the increasing temperature. A linear curve fitting to the experimental data shows an average slope of $15 \mathrm{pm} /{ }^{\circ} \mathrm{C}$, which is similar to the temperature sensitivity $\left(16 \mathrm{pm} /{ }^{\circ} \mathrm{C}\right.$ in the temperature range of $200-1100{ }^{\circ} \mathrm{C}$ ) of the surface relief FBG sensor reported in Ref. 8. In the experiments conducted when the temperature reached $800{ }^{\circ} \mathrm{C}$, we noticed that the shape of the spectrum started to change and the changes were not reversible when the temperature was reduced. This could be caused by the contamination of the end surface of the MMF or by the core dopant diffusion of the SMF at elevated temperatures, ${ }^{11}$ and will be further investigated.

In conclusion, we have proposed and demonstrated a fiber-optic temperature sensor by using the interference of selective higher-order modes in circular optical fibers. Similar to FBG-based temperature sensors, the proposed sensing

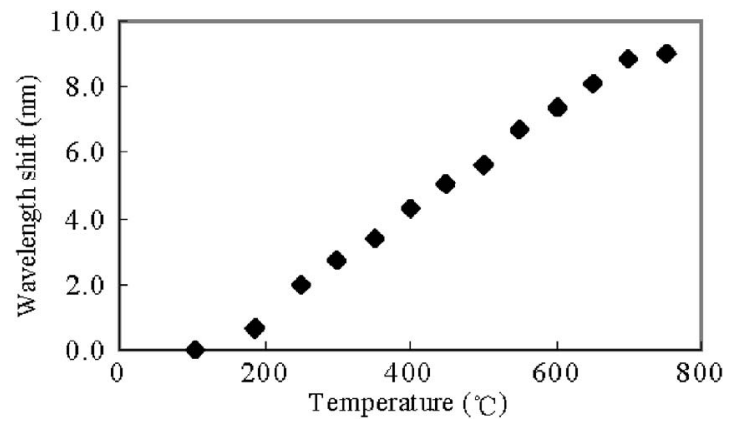

FIG. 3, Measured wavelength shifts under different temperatures. 
technique has advantages such as wavelength encoding and wavelength multiplexing capabilities. Compared to other fiber-optic temperature sensors, the sensors reported here feature an extremely simple structure and fabrication process, and hence a very low cost. Moreover, the sensors have the potential for high-temperature applications. Our tests on the developed sensors show that the wavelength shift varies linearly with the temperature changes in the range of $100-750{ }^{\circ} \mathrm{C}$ with an average temperature sensitivity of $15 \mathrm{pm} /{ }^{\circ} \mathrm{C}$. In principle, higher temperatures could be measured if the end surface of the MMF can be properly processed and fibers with more stable waveguide characteristics at high temperatures are used to fabricate the sensor. With the current design, a cleaved surface was used as a reflection mirror, which provided very low reflection. In order to increase the reflectivity, we are now testing fiber mirrors formed by coating the MMF end surface with aluminum.

This work was supported in part by Natural Science Foundation of China (Grant No. 60578054), Tianjin Munici- pal Commission of Education, and Australian Research Council.

${ }^{1}$ R. M. Measures, Structural Monitoring with Fiber Optic Technology (Academic, San Diego, CA, 2001), pp. 369-449.

${ }^{2}$ A. Othonos and K. Kalli, Fiber Bragg Gratings: Fundamentals and Applications in Telecommunications and Sensing (Artech House, Boston, MA, 1999), pp. 98-140.

${ }^{3}$ K. O. Hill and G. Meltz, J. Lightwave Technol. 15, 1263 (1997).

${ }^{4}$ A. D. Kersey, M. A. Davis, H. J. Patrick, M. LeBlanc, K. P. Koo, C. G. Askins, M. A. Putnam, and E. J. Friebele, J. Lightwave Technol. 15, 1442 (1997).

${ }^{5}$ S. R. Baker, H. N. Rourke, V. Baker, and D. Goodchild, J. Lightwave Technol. 15, 1470 (1997).

${ }^{6}$ S. Kannan, J. Z. Y. Guo, and P. J. Lemaire, J. Lightwave Technol. 15, 1478 (1997).

${ }^{7}$ L. Dong and W. F. Liu, Appl. Opt. 36, 8222 (1997).

${ }^{8}$ T. L. Lowder, K. H. Smith, B. L. Ipson, A. R. Hawkins, R. H. Selfridge, and S. M. Schultz, IEEE Photonics Technol. Lett. 17, 1926 (2005).

${ }^{9}$ W. S. Mohammed, A. Mehta, and E. G. Johnson, J. Lightwave Technol. 22, 469 (2004).

${ }^{10}$ D. Marcuse, J. Lightwave Technol. LT-5, 125 (1987).

${ }^{11}$ M. N. McLandrich, Electron. Lett. 24, 8 (1988). 